\title{
The effect of emerging therapies on cardiopulmonary disease in Duchenne muscular dystrophy
}

\author{
Doug McKim ${ }^{1}$, Timothy Cripe $^{2}$, and Linda Cripe $^{3}$ \\ ${ }^{1}$ Ottawa Hospital Research Institute \\ ${ }^{2}$ Nationwide Children's Hospital \\ ${ }^{3}$ Nationwide Children's Hospital
}

August 4, 2020

\begin{abstract}
Gene therapy is an attractive approach being intensively studied to prevent muscle deterioration in patients with Duchenne muscular dystrophy. While clinical trials are only in early stages, initial reports are promising for its effects on ambulation. Cardiopulmonary failure, however, is the most common cause of mortality in DMD patients, and little is known regarding the prospects for gene therapy on alleviating DMD-associated cardiomyopathy and respiratory failure. Here we review current knowledge regarding effects of gene therapy on DMD cardiomyopathy and discuss respiratory endpoints that should be considered as outcome measures in future clinical trials.
\end{abstract}

\section{Introduction}

Duchenne muscular dystrophy (DMD) is a rare X-linked genetic disorder that results from out of frame mutations in the dystrophin gene. The incidence is believed to be 1 in 5,000 male births ${ }^{1}$; however, it is now appreciated that female carriers can also manifest the disease. Dystrophin is a critical protein involved in membrane stabilization by anchoring the inner surface of the sarcolemma to F-actin ${ }^{2}$. Disruption of the protein results in both progressive skeletal muscle disease as well as a cardiomyopathy. Modern advancements in respiratory therapies have significantly impacted survival with the cardiomyopathy now considered the leading cause of mortality. While the focus historically has been largely on the impact of therapies on skeletal muscle disease, with the advent of emerging molecular and genetic therapies, clinical trials will need to focus on understanding the impact not only on skeletal muscle function but also on cardiopulmonary disease. The failure of many trials to include cardiac and even sometimes respiratory endpoints has impaired our ability to understand the impact of contemporary therapies on the heart and lungs.

If there is not a family history of the disease, children tend to be diagnosed within the first few years of life when they fail to meet gross motor milestones. Clinical cardiac dysfunction tends not to occur later in the disease, but we now know that the myocardium is impacted early in the disease. In fact, EGC's taken in the newborn and young child are often abnormal and show evidence of left ventricular hypertrophy. Furthermore, cardiac magnetic resonance imaging (CMR) has allowed us an important window into what is occurring in the heart of the young DMD patient. Abnormalities of myocardial strain are noted before the onset of the development of myocardial fibrosis or declining function. Traditional heart failure medications are the only avenue of treatment at this time. Left ventricular assist devices (LVAD) and cardiac transplantation are treatments that have been utilized in isolated instances.

Early reports of new advances in gene therapy appear promising for delaying loss of ambulation and improving quality of life in children with DMD ${ }^{3}$. The impact of gene therapy on cardiopulmonary outcomes, howver, 
is unknown. Herein we review preclinical data regarding the potential for gene therapy to ameliorate DMDassociated cardiomyopathy and review the landscape of current DMD gene therapy efforts, including gene replacement, gene modulation, and gene editing. Because even less is known about the effects of gene therapy on lung function, we focus our discussion on various pulmonary endpoints that should be considered as potential outcome measures in future trials of DMD gene therapy.

\section{Gene Therapy and the DMD Heart}

The promise of gene therapy is to restore expression of a normal protein in a cell containing a genetically deficient or defective version. In that sense, gene therapy raises the possibility of a cure for DMD, not only for skeletal muscle but also for the heart. Strictly speaking, gene therapy is use of nucleic acid, either DNA or RNA (or both) to alter the function of a cell. A variety of approaches have been tried to carry nucleic acid into a cell, including direct injection of DNA (so-called "naked" DNA), DNA transfected into a cell by compacting it with a glycoprotein/polycation scaffolding, encapsulated in liposomes that fuse with the cell membrane, or most commonly packed in a virus shell. Viruses have evolved to efficiently enter cells and transmit DNA to the nucleus, and many can be "gutted" of many or all of their normal genes to make room for transferring in new genes. The new gene is referred to as a "transgene," virus-mediated entry into a cell is termed "transduction," and the virus carrying the transgene is called a "vector." While many different viruses have been used for transduction, a small member of the parvovirus family, adeno-associated virus (AAV), is most commonly used due to its safety profile (it is not associated with any disease), efficiency of transduction, widespread biodistribution following intravenous delivery, and ease of manipulation. Whereas the normal "wild type" adeno-associated virus tends to integrate its DNA into a specific locus on chromosome 19 , gutted versions containing a transgene persist in the nucleus as circular episomes, separate from the cellular chromosome. This lack of integration markedly reduces the "genotoxicity," meaning the chance for causing genetic mutations in cellular genes, but it also increases the chance the new gene could be "lost," since it is not permanently incorporated into the cellular genome.

Critical to the success of gene therapy is ensuring adequate expression in the diseased cell, meaning skeletal and heart muscle cells in patients with DMD. There are two major determinants of adequate expression, the AAV serotype and promoter. The serotype (a word originally used to define related viruses distinct enough not to show cross reactivity with immune serum) determines the amino acid sequence of the outer shell of the virus, which contains the "key" to enter a cell; different serotypes bind to different cell surface receptors to gain entry. While most but not all naturally occurring AAV serotypes enter skeletal muscle cells, only a subset also enter heart muscle cells. Each serotype has its own unique biodistribution, as do many new synthetic serotypes being created and explored to preferentially transduce specific tissues. A seemingly infinite variety of capsid variations are possible, and finding those best suited for DMD is underway ${ }^{4}$. No virus has yet been discovered or created that is completely specific for a given cell type, and most viruses transduce liver cells after given intravenously due to features of circulation and liver uptake, in addition to other tissues. Once the virus binds and enters a cell, the second determinant regarding expression is the activity of the enhancer/promoter in that cell. There are many well-known naturally occurring strong enhancer/promoters, such as from a cytomegalovirus gene, the elongation factor 1a gene, and chicken $\beta$-actin, as well as many synthetic and hybrid versions, that are capable of activating gene expression across a wide variety of cell types. While these promoter/enhancers might work well in muscle, they may also express the transgene, in this case a small form of dystrophin, in cells that don't normally express it, which could cause harm. Thus, gene therapy vectors for DMD use enhancers/promoters derived from muscle-specific genes such as muscle creatine kinase, desmin, and myosin heavy chain, in order to restrict expression to muscle cells. Taken together, in order for gene therapy to work in the DMD heart, it must be comprised of a vector serotype that transduces cardiac muscle cells and an enhancer/promoter that is active or "on" in those cells.

The choice and structure of the transgene is also an important consideration, especially in DMD. Because the full-length dystrophin protein is 3,685 amino acids, it requires 11,595 DNA nucleotide bases to encode it (3 bases per amino acid). AAV shells are quite small, about 70 nanometers, and they can only package approximately 4,700 bases of DNA. From this vantage point, some viruses with large packaging capacities such 
as herpes simplex virus might seem more attractive, but many such larger virus types do not transduce muscle cells well. The main solution employed so far to solve this size dilemma has been to use a shortened version of dystrophin. Much of the dystrophin protein within the so-called rod domain is comprised of numerous repeats, which can be deleted to fit into AAV. While these so-called mini-dystrophin (akin to dystrophin in Becker patients) or micro-dystrophin (with nearly all repeats deleted) proteins are not normal, they are sufficient to restore significant function to muscle cells. Such an approach markedly improved cardiomyopathy in aged mdx mice, resulting in improvements on electrocardiography, hemodynamic measurements, and prevented dobutamine-stress induced cardiac death ${ }^{5}$.

Ongoing clinical development of gene therapy for DMD is currently being spear-headed by three main companies. Sarepta is using an AAV serotype derived from rhesus monkeys called rh74. An advantage of this serotype due to it being a monkey virus is that, ostensibly, fewer people have pre-existing immunity to AAVrh74 compared to human-derived AAV serotypes. Their micro-dystrophin gene is driven by an engineered hybrid promoter, MHCK7, derived by combining sequences from the creatine kinase and alphamyosin heavy chain complex promoters and shown to have high expression in both cardiac and skeletal muscle cells $^{6}$. Thus, it is anticipated this virus will be effective in treating the heart. Similarly, Solid Biosciences uses an AAV9 serotype which enters skeletal and cardiac cells as well as a variety of other tissues, and a muscle creatine kinase 8 promoter, which also shows robust cardiac expression in preclinical studies. In contrast, while the serotype being used by Pfizer is also an AAV9 serotype, their mini-dystrophin gene is driven by the human creatine kinase promoter that for which some data suggest it is not particularly active in cardiac cells. This construct may do well in skeletal muscle, but whether it significantly ameliorates DMD-associated cardiomyopathy is unclear.

As an alternative to replacing the defective dystrophin gene with a shortened version, another approach is to express a gene to help the disease muscle cell in other ways. The GALGT2 gene encodes glycosyltransferase that increases expression of a variety of proteins that can improve muscle cell function such as utrophin, plectin1, agrin, several laminins and integrin. Indeed, AAV-mediated gene transfer of GALGT2 improves not only skeletal muscle pathology in the mdx mouse but also is very effective at improving cardiac function ${ }^{7}$ and is currently in clinical trials (https://clinicaltrials.gov/ct2/show/NCT03333590).

Some dystrophin mutations are amenable to exon skipping; that is, their alterations or deletions are contained within a single exon that essentially derails the rest of the protein by changing the reading frame and resulting in a premature stop codon. In such cases, antisense oligonucleotides designed to specifically interfere with splicing of that particular exon can cause it to be excluded from the final mRNA during splicing, restoring the reading frame and thus the remainder of the protein. In fact, a chemically stabilized antisense oligonucleotide (so-called "morpholino") specific for exon 51 is FDA approved for patients with mutations in that exon, and other antisense molecules are being developed for other exons. The major limitation of this approach is achieving sufficient quantities to enough muscle nuclei to have a broad and high impact on restoring dystrophin expression, though conjugation of the antisense with peptides may improve delivery. Another approach to overcome this limitation is to place the antisense sequence in a U7 small nuclear RNA expressed in an AAV, again leveraging the virus biology to achieve high levels in the muscle nucleus. Interestingly, the combination of both the peptide-conjugated morpholino together with AAV-U7 appears even better than either approach alone ${ }^{8}$.

Due to recent biotechnological advances, over the past few years the definition of gene therapy has expanded from one that is primarily about "therapy using nuclei acids or genes," such as AAV-insertion of a functional gene into the nucleus or anti-sense oligonucleotides to skip an exon as described above, to one that encompasses "therapy OF genes." By leveraging and adopting a bacterial system of proteins and template or "guide" RNAs, we can now precisely edit genes in situ ; that is, we can make deletions or insertions into the cellular genome at specific locations based on the unique DNA sequence. Again a nuclei acid is used as a therapeutic, as the hybridization to DNA, or the "matching" of the guide RNA sequence to the DNA, determines where in the DNA to cut and/or insert. Critical to the process is the bacterial protein as well, known as the clustered regularly interspaced short palindromic repeats (CRISPR)-associated protein (Cas). There 
are several Cas family members, with Cas9 the most commonly one in use. This new capability is rapidly transforming our vision of what diseases might someday be treatable with gene therapy, including DMD. In fact, encouraging proof-of-principle studies in mice have been published. For example, in the mdx mouse that contains a mutation in exon 23 of dystrophin, CRISPR/Cas9 was successful used to delete the mutant exon, restore the reading frame, and partially restore dystrophin expression, resulting in improved function of both skeletal and cardiac muscle ${ }^{9,10}$. While promising, there remain a number of barriers to clinical use of CRISPR/Cas9 technology. The follow-up study of those mice showed persistence, long-term expression one year later, but detailed analysis also reviewed immunity to the bacterial Cas9 protein and unintended "off target" gene editing ${ }^{11}$. The latter has been a concern in the field since its inception, and exactly how precisely we can edit the genome without inducing other mutations that either could be damaging to the cell or cause other diseases such as cancer is unknown. Another potential barrier to CRISPR/Cas9 use in humans was recently uncovered: pre-existing immunity to bacterial Cas9, given humans are colonized with a microbiome, which in a mouse model destroyed cells edited with CRISPR/Cas9 due to anti-Cas9 immunity 12 .

\section{Respiratory Outcome Measures for Trials of Emerging Therapies in Duchenne Muscular Dys- trophy}

While the goal of gene modifying and molecular therapies for DMD is to improve survival, the duration of prospective clinical trials are too limited to be capable of demonstrating an impact on this outcome. As such, surrogate measures of respiratory function which correlate with survival and quality of life (QoL) are necessary. Measures must also be sufficiently sensitive to change within the population for whom the therapeutic effects can be the most meaningful, those younger individuals with DMD.

In order to design clinical trials of novel therapies and assess their impacts on respiratory function, it is necessary to appreciate the natural history of the time course and evolution of respiratory muscle and pulmonary function in DMD as well as the impact that standard therapies may have on that evolution. Furthermore, and perhaps more importantly, multiple individuals with identical genetic features may have significantly different trajectories for cardiopulmonary function. As such, it will be necessary to stratify randomization by phenotypic behavior in order to more accurately evaluate the effect of emerging therapies.

\section{Natural history of respiratory function in DMD}

A major measure of lung function is absolute vital capacity (VC), which rises from age 7 and usually peaks between 12 and 14 years and then declines due to progressive muscle weakness. While absolute VC continues to rise, the percentage of ideal or predicted $\mathrm{VC}$ (VC\%pred) declines in a linear fashion reaching about $80 \%$ predicted at age $10^{13}$. Hence, gradually falling VC\%pred values include absolute values which occur before, at and following peak VC. Some investigators suggest that the identification of absolute values before, at and following peak VC may be best to assess the effect of interventions ${ }^{14}$. In a large cohort study the VC of untreated individuals peaked at 1.85 liters at age 12 to $13 \mathrm{yrs}$ and began to decline when VC\%pred fell to $60-70 \%{ }^{15}$. Rates of decline of VC\%pred per year have varied in the literature between as little as $5 \%$ (ages 5 to $24 \mathrm{yrs})^{16}$ to as much as $10.7 \%$ (ages 12 to $15 \mathrm{yrs)}{ }^{17}$. Following peak VC, absolute decline in $\mathrm{ml} / \mathrm{yr}$ varies between $118{ }^{14}$ and $180 \mathrm{mls} / \mathrm{yr}{ }^{18}$ with subsequently both $\mathrm{FVC}$ and $\mathrm{VC} \%$ pred reaching an asymptotic floor after 20 years of age $\mathrm{e}^{14-16,19,20}$. Without ventilatory support, a more favorable prognosis is associated with a higher, later peak and a more gradual decline in VC ${ }^{21}$. Consensus guidelines recommend lung volume recruitment strategies at VC $60 \%$ pred, mechanically assisted coughing at 50\%pred and NIV below $50 \%$ pred $^{22}$. A VC of less than 1 liter has been associated with a 5 year survival of only $8 \%$ without respiratory supports ${ }^{18}$. At VCs below a liter $(680 \mathrm{cc})$ up to 24 hour respiratory support, preferably noninvasively, may be required. As such, disease modifying therapies have the goal of increasing and delaying the peak VC and reducing the post-plateau rate of decline. The more or less linear decline of VC\%pred as an outcome measure may not be as sensitive to change as a result of interventions, as absolute $\mathrm{VC}^{13}$.

Phenotypic differences in pulmonary function and therefore clinical outcomes have been recognized for years. Phillips identified a longer surviving population who experienced peak VC at a median of 2 years later, 13 
vs. 15 years and a slower yearly decline in VC\%pred, $6 \%$ vs, $7.8 \%{ }^{18}$ and other investigators have divided DMD populations in to cohorts with different outcomes and survival related to the number of mutations upstream to exon $30^{23}$. Even within the same genotype heterogeneity exists and needs to be considered in the evaluation of emerging therapies ${ }^{23}$.

\section{Effect of standard therapies on pulmonary functions and clinical outcomes}

Glucocorticoid (GC) therapy is well established to help delay and increase the maximum VC plateau though this is then followed by the same rate of loss. McDonald found that compared to untreated individuals whose VC peaked at 1.85 liters between ages of 12.0 to 12.9 yrs, those treated with GCs had a peak median FVC of 2.03 liters at age 17.0 to 17.9 years, both increasing and delaying the maximum VC. As well, the age at which VC fell below 1 liter was delayed from 20.0-20.9 until 23.0-23.9 years ${ }^{16}$.

In addition to regular monitoring of pulmonary function and gas exchange, international guidelines recommend preventive and supportive respiratory therapies for individuals with DMD; lung volume recruitment strategies, manual and mechanical cough assistance and noninvasive ventilation (NIV) ${ }^{22,24}$. A number of these therapies have effects on respiratory function and its evolution.

Lung Volume Recruitment (LVR). LVR, the active or passive stacking of breaths with a resuscitation bag or mouthpiece ventilator in order to achieve a maximum insufflation capacity (MIC) has been demonstrated to have a beneficial effect, not only on cough flows but on lung mechanics. There is evidence for short term improvement in respiratory system compliance and observational evidence for a longer-term effect on important measures such as rate of loss of $\mathrm{VC}$ as a result of LVR.

Molgat-Seon demonstrated that respiratory system compliance can be significantly improved for an hour after a session of $\mathrm{LVR}^{25}$ and Chiou showed that the peak/plateau VC can be delayed by 5 years and the post-peak rate of decline significantly decreased ${ }^{15}$. The mechanism for improvement in loss of VC is most likely related to the establishment and maintenance of a maximum insufflation capacity (MIC). The MIC-VC difference and the resulting peak cough flow are the measures of effectiveness of LVR. In a small retrospective study, McKim demonstrated that the rate of decline of VC \%pred after age 18 can be reduced to $0.5 \%$ per year $^{26}$ compared to a recent cohort of 47 DMD subjects not using GCs with mean age of $17.5 \pm 4.7$ years and a mean rate of $\mathrm{FVC} \% \mathrm{p}$ decline of $4.68 \% / \mathrm{yr}{ }^{27}$. In addition, the MIC-VC difference can be maintained for over 8 years at a level sufficient to achieve effective cough flows ${ }^{28}$.

Noninvasive ventilation (NIV). NIV has provided the most profound effect on survival in DMD, from the late teens to at least the late 20 's, even into the 40 s in the presence of favorable cardiac function and 24 hour, continuous NIV/mouthpiece ventilation, in spite of little or no measurable pulmonary function ${ }^{29-31}$. Initiation of NIV can be considered, in pharmacotherapy trials, as an important outcome to be delayed as a consequence of improved muscle function. However, if necessary and initiated, NIV can have important and significant effects on sleep quality, quality of life and, of course on survival, even with no measurable VC 32,33 .

A small number of publications indicate an improvement in the rate of loss of VC in other progressive neuromuscular conditions such as ALS, as a result of NIV initiation ${ }^{34}$. The introduction of daytime NIV (mouthpiece ventilation) in DMD patients has been demonstrated to increase the endurance of fatigued inspiratory muscles ${ }^{35}$. In a 3-month prospective controlled trial, Schonhofer et al. demonstrated a significant increase in inspiratory threshold load endurance time of $278+/-269 \%$ indicating improved inspiratory muscle function ${ }^{36}$.

Nickol et al ${ }^{37}$ studied 12 subjects with neuromuscular disease (NMD, not specified), baseline mean TLC $62 \%$ pred and another 8 with scoliosis, after three months of NIV and found a significant increase in maximum expired pressure (MEP) and hypercapneic ventilatory response (HCVR) as well as a trend toward increases in inspiratory strength indicated by more negative maximum inspiratory esophageal (SnPes), sniff nasal inspiratory pressure (SNIP). No significant increase was noted in maximum inspiratory pressure (MIP). Given the small number of subjects, lack of significance in respiratory muscle function could have been as a 
result of a beta error.

Mechanical In-Exsufflation (MI-E). Mechanical cough assistance, generally administered through a noninvasive mask at pressures of $+/-40 \mathrm{cmH} 2 \mathrm{O}$ or greater, has not only been associated with reduced hospitalizations and intubations due to more effective airway clearance ${ }^{38}$ but has also been demonstrated to improve vital capacity and lung mechanics.

In a study of 21 patients with NMD, 10 of whom had DMD (VC 11\%Pred, all using NIV) Stehling et al demonstrated an average improvement of $28 \%$ in $\mathrm{VC}$, from $0.48+/-0.15$ to $0.59+/-0.25$ liters after one year of twice daily MI-E therapy ${ }^{39}$. As average MI-E pressures were only $+25.1+5.3$ and $-25.6+5.7 \mathrm{cmH} 2 \mathrm{O}$, a greater effect on VC might be anticipated with pressures more commonly utilized clinically. Although these were older individuals (age 20.6 +/- 3.9 yrs) with severe respiratory muscle weakness, regular use of MI-E clearly has the capacity to improve measures such as $\mathrm{VC}$.

Although data are limited, taken together, these observations suggest that standard therapies recommended by international guidelines may have a significant effect on some of the primary respiratory outcomes being evaluated in studies of molecular and gene therapies and as such, respiratory interventions should be well documented and accounted for in randomization and evaluation.

\section{Effect of phenotypic heterogeneity}

Another important factor potentially confounding the interpretation of aggregate results of intervention studies on respiratory outcomes is phenotypic heterogeneity. This variability has been recognized for years ${ }^{18}$ and can manifest as markedly different timing and value maximum VC as well as rate of decline. Neve, in a study of 33 CS naïve DMD subjects identified subphenotypes requiring NIV either prior to, or after age $17{ }^{40}$. The former was identified by an earlier (age 11.5 to 13) and lower peak VC and SNIP and more rapid decline. In a study using the STRIDE nonsense mutation (nmDMD) cohort, investigators found no correlation in 181 patients between disease severity and exon location of the nonsense mutation. There was a large degree of phenotypic variability despite of having identical dystrophin mutations ${ }^{41}$. Birnkrant has identified highly discordant cardiopulmonary function in brothers with DMD and identical dystrophin mutations and identified 8 of 15 patients $(53 \%)$ with a shared genotype who had discordant cardiopulmonary function $^{23,42}$. Clinical manifestations were not related to the dystrophin mutation. In a cohort of over 200 patients, Magri, as well, noted the absence of correlation of pulmonary, cardiac or motor phenotype with dystrophin genotype, with the exception of neurocognitive function being related to more distal mutations 43 .

Being unable to predict the natural evolution of cardiopulmonary function in a portion of the population despite common genotype could pose a significant confounding issue when assessing the impact of emerging therapies in DMD. Rather than being the true treatment effect, such beneficial or adverse observations might simply be due to more favorable or unfavorable phenotype.

\section{Results of current gene modifying and molecular therapies}

There is a number of recent studies of novel therapies for DMD (Ataluren, Indebadone and Etiplersin) which include either primary or secondary respiratory outcomes.

Ataluren is an oral treatment for patients with nonsense mutation DMD (nmDMD), designed to facilitate ribosomal readthrough of an in-frame premature stop codon, increasing production of full-length dystrophin protein ${ }^{44}$. Propensity matching, using age at first symptoms, age at first use and duration of steroid use as well as time-to-event motor, pulmonary and cardiac features, was employed in order to match patients in the Strategic Targeting of Registries and International Database of Excellence (STRIDE) to individuals in an historical registry, the Cooperative International Neuromuscular Research Group Duchenne Natural History Study (CINRG DNHS) ${ }^{41}$. Individuals were matched according to age at first symptoms, age at first use and duration of steroid use, as well as time-to-event motor, pulmonary and cardiac features. Mercuri et al. demonstrated significant impacts on measures of skeletal muscle function (standing, stair climbing, timed motor tasks) in nonsense mutation DMD (nmDMD) but for a number of reasons, including the insensitivity 
of the outcome measures used ( $\mathrm{VC} \%$ pred), the relative youth of the treated subjects compared to controls and the duration of follow up, no significant impact on respiratory outcomes was identified.

In a publication describing the results of three studies of Eteplirsin, Khan ${ }^{45}$ found a significant difference in the rate of decline of $\mathrm{VC} \%$ pred in $74 \mathrm{DMD}$ patients; 2.19 to $3.79 \%$ per year for Eteplirsin compared to 5.56 to $6 \%$ per year for the three CINRG DNHS control groups. However, the study group 201/202, randomized initially to placebo vs. 30 or $50 \mathrm{mg}$ Eteplirsen, was only 1.5 years younger and had the greatest improvement in VC decline. However, there was a considerably higher baseline \%pred VC of $96.9 \%$ for the Eteplirsin treated subjects vs. at best, $79.6 \%$ for the controls. Interestingly, no data were presented for the placebo-controlled portion of the study. The authors concluded that the results were "meaningful" and "will likely impact both the quality of life and the duration of life in patients with DMD."

In a Phase II randomized placebo-controlled study in 21 DMD boys at age $8-16$ years, Buyse ${ }^{46}$ found that idebenone, a short-chain benzoquinone, antioxidant and inhibitor of lipid peroxidation, at a dose of 450 $\mathrm{mg} / \mathrm{d}$ had a significant effect on their primary outcome, peak expired flow (PEF) but only in steroid naïve subjects. PEF, in the absence of air flow limitation, is not only a composite reflection of VC, MIP and MEP but also bears a marked clinical significance in terms of cough effectiveness and airway clearance in patients with NMD. In DMD, absolute PEF remains quite stable during the first and second decade but PEF\%pred declines linearly from age 7 or $8^{13}$. Not surprisingly, values of PEF\%p correlated well with the percent predicted values for MIP and FVC. Idebenone was associated with an $8.0 \%$ improvement in PEF\%pred, while those on placebo declined by $12.3 \%(\mathrm{p}<0.05)$ over 12 months. Indebenone did not significantly affect the rate of decline in steroid treated subjects.

A subsequent, one-year phase 3 placebo-controlled trial for DMD subjects age 10-18 yrs who were not using steroids, was also reported. Subjects were randomized to Idebenone $900 \mathrm{mg}$ daily vs. placebo with the primary outcome, again being PEF\%pred ${ }^{47}$. In the intention to treat analysis, the one-year change in PEF\%pred from baseline was -2.57 vs. $-8.84, \mathrm{p}<0.0001$. The Idebenone group was 1.5 yrs younger and although post hoc analysis did not indicate that age had an effect, younger patients did seem to benefit more, possibly because their VC (absolute) values were still rising. Observations were not influenced by past steroid use. No significant differences were found in the change from baseline for MIP, MEP or peak cough flow. The authors also noted fewer patients in the treated group reaching milestones of VC $(1 \mathrm{~L})$ and peak cough flow $(160 \mathrm{~L} / \mathrm{min})$ suggesting that delaying these thresholds might result in less morbidity and mortality. Avoiding the need for ventilatory support and airway clearance for a period of time might indeed be a useful outcome but again, noninvasive ventilation when needed improves sleep quality and QoL and it is well established that DMD patients can survive for decades with vital capacities well below $1 \mathrm{~L}$, even zero $(23,28,39)$.

Taken together, these studies do suggest a modest impact of emerging therapies on respiratory function decline in individuals with DMD. Whether or not an improvement in health-related quality of life can be demonstrated remains to be seen. As long as noninvasive ventilation and airway clearance strategies are properly employed, survival is unlikely to be impacted by additional treatments. Mortality continues to be defined by favorable or unfavorable cardiac function, not respiratory decline ${ }^{48}$.

\section{Respiratory Outcome Measures to Consider in Future Studies}

Given the limitations of pulmonary function measures in a number of studies to date, more sensitive measures are needed which account for the natural evolution of respiratory function at different ages.

Maximum inspiratory pressure (MIP)

Based on the pressure/volume relationship of the respiratory system and the reserve present in respiratory muscles, it is accepted that MIP is a much more sensitive, earlier measure of respiratory muscle impairment than VC ${ }^{49,50}$, although VC has been widely used in clinical management and study outcomes. Indeed, in younger patients, VC is still rising in to early teen years. In a review paper, Schoser found MIP included as a secondary outcome in one published ${ }^{46}$ and three ongoing trials in DMD. In the Idebenone trial ${ }^{46}$ MIP improved, though not significantly compared to a decline in the placebo group. There is however contradiction 
in published results. Neve studied 33 steroid naïve DMD age 11 (5-16.7 yrs) and found no significant change in absolute MIP values ${ }^{40}$ while Gayraud found a declining MIP in a similar age group $(9.1+/-1$ to $16+/$ $1.4 \mathrm{yrs}$ ) with \%pred MIP being only $67 \%$ at first measurement ${ }^{17}$. MIP might, then, be superior to some outcome measures for interventions directed at young patients with DMD in whom VC is relatively well preserved.

\section{Sniff nasal inspiratory pressure (SNIP)}

In the absence of much change in MIP, Neve concluded that sniff nasal inspiratory pressure (SNIP) was a more sensitive measure of weakness ${ }^{40}$. In a 3-year study of 33 steroid-naïve, 5-20-year-old DMD patients, SNIP measurements were found to be reliable. SNIP and VC increased until 10.5 and 12.5 years of age, respectively, and then declined. SNIP identified respiratory muscle impairment earlier (at 10.5 years) than VC (at 12.5 years). Others have found measurements of SNIP to have less variability and to be performed consistently in children as young as 5 yrs ${ }^{51}$. Standardized performance of SNIP measurements is not entirely agreed upon and a recent publication, including about $20 \%$ muscular dystrophy patients, noted that occluding the opposite nostril from that with the pressure monitor resulted in consistently higher values ${ }^{52}$.

Abdominal/Rib Cage contribution to tidal volume

In a retrospective but elegant, 7-year study of 115 individuals with DMD, 6-24 yrs old, with 574 visits, LoMauro ${ }^{13}$ employed nonlinear regression model analysis and presented the evolution of mean curves of spirometry, lung volumes, spontaneous breathing and thoraco-abdominal pattern, measured by optoelectronic plethysmography. Although more appropriate for older subjects, measures of respiratory pattern have the advantage of requiring no cooperation or effort. During spontaneous breathing it was determined that the abdominal contribution to tidal breathing was reduced at 14.8 yrs., the tidal volume was reduced at 17.3 yrs., the minute ventilation was reduced at 18.1 yrs. and minute ventilation at 22 yrs. Spontaneous respiratory patterns are not commonly studied but may have a role to play in assessing the effect of therapeutic interventions, particularly for those who are unable to cooperate with pulmonary functions measurements such as those under 6 yrs. or with severe weakness or cognitive delay

\section{Challenges in Trial design}

As discussed above, the interpretation of the impact of emerging therapies on respiratory function using aggregate data is confounded by phenotypic variability where it may not be possible to determine if a subject is evolving naturally with favorable or unfavorable respiratory function. As well, the age group commonly evaluated combines subjects whose VC may be rising with others whose VC is declining. When true randomization is not possible, attempts to accommodate for this have included propensity matching as in the ataluren study ${ }^{41}$. However, propensity score matching can only adjust for measured covariates, and one cannot be assured that all confounding factors have been eliminated ${ }^{52}$.

Becuase of the somewhat unpredictable evolution of respiratory function in any given individual which may confound study results for a treatment intervention, consideration might be given to an $\mathrm{N}$ of 1 , crossover design where subjects are compared with themselves under control and experimental conditions and crossed over at intervals from one to the other ${ }^{53}$. The duration of recent studies and the suggestion of improvements due to treatment as early as 26 weeks ${ }^{47}$, indicate that these investigations might lend themselves to such an $\mathrm{N}$ of 1 design, e.g. randomization to 6 to 12 months of intervention or placebo, followed by crossover for the remaining 6 to 12 months. Such a design would help address the issues with regard to randomization and phenotypic variability.

Future studies should employ outcome measures which take in to account the dynamic of respiratory function evolution at different ages and which are sensitive to change. A number of parameters should be followed including those which are sensitive to respiratory muscle strength (MIP, SNIP) and absolute VC which, particularly in an $\mathrm{N}$ of 1 design, would account for individual variations in age at maximal $\mathrm{VC}$ and rate of decline. The utilization and impact of standard therapies must also be considered in fully understanding the effect of emerging therapies. 


\section{References Cited}

1. Mendell JR, Shilling C, Leslie ND, et al. Evidence-based path to newborn screening for Duchenne muscular dystrophy. Ann Neurol.2012;71(3):304-313.

2. Nowak KJ, Davies KE. Duchenne muscular dystrophy and dystrophin: pathogenesis and opportunities for treatment. EMBO Rep.2004;5(9):872-876.

3. Mendell J, Sahenk Z, Lehman K, et al. Assessment of Systemic Delivery of rAAVrh74.MHCK7.microdystrophin in Children With Duchenne Muscular Dystrophy: A Nonrandomized Controlled Trial. JAMA Neurol.2020;Published online June 15, 2020. doi:10.1001/jamaneurol.2020.1484.

4. Nance ME, Duan D. Perspective on Adeno-Associated Virus Capsid Modification for Duchenne Muscular Dystrophy Gene Therapy. Hum Gene Ther. 2015;26(12):786-800.

5. Bostick B, Shin JH, Yue Y, Duan D. AAV-microdystrophin therapy improves cardiac performance in aged female mdx mice. Mol Ther.2011;19(10):1826-1832.

6. Salva MZ, Himeda CL, Tai PW, et al. Design of tissue-specific regulatory cassettes for high-level rAAVmediated expression in skeletal and cardiac muscle. Mol Ther. 2007;15(2):320-329.

7. Xu R, Jia Y, Zygmunt DA, Martin PT. rAAVrh74.MCK.GALGT2 Protects against Loss of Hemodynamic Function in the Aging mdx Mouse Heart.Mol Ther. 2019;27(3):636-649.

8. Forand A, Muchir A, Mougenot N, et al. Combined Treatment with Peptide-Conjugated Phosphorodiamidate Morpholino Oligomer-PPMO and AAV-U7 Rescues the Severe DMD Phenotype in Mice. Mol Ther Methods Clin Dev. 2020;17:695-708.

9. Nelson CE, Hakim CH, Ousterout DG, et al. In vivo genome editing improves muscle function in a mouse model of Duchenne muscular dystrophy. Science. 2016;351(6271):403-407.

10. Hakim CH, Wasala NB, Nelson CE, et al. AAV CRISPR editing rescues cardiac and muscle function for 18 months in dystrophic mice. JCI Insight. 2018;3(23).

11. Nelson CE, Wu Y, Gemberling MP, et al. Long-term evaluation of AAV-CRISPR genome editing for Duchenne muscular dystrophy. Nat Med. 2019;25(3):427-432.

12. Li A, Tanner M, Lee C, et al. AAV-CRISPR gene editing is negated by pre-existing immunity to Cas9. Mol Ther. 2020;28(6):1432-1441.

13. LoMauro A, Romei M, Gandossini S, et al. Evolution of respiratory function in Duchenne muscular dystrophy from childhood to adulthood.Eur Respir J. 2018;51(2).

14. Chiou M, Bach JR, Jethani L, Gallagher MF. Active lung volume recruitment to preserve vital capacity in Duchenne muscular dystrophy. J Rehabil Med. 2017;49(1):49-53.

15. McDonald CM, Gordish-Dressman H, Henricson EK, et al. Longitudinal pulmonary function testing outcome measures in Duchenne muscular dystrophy: Long-term natural history with and without glucocorticoids. Neuromuscul Disord. 2018;28(11):897-909.

16. Mayer OH, Finkel RS, Rummey C, et al. Characterization of pulmonary function in Duchenne Muscular Dystrophy. Pediatr Pulmonol.2015;50(5):487-494.

17. Gayraud J, Ramonatxo M, Rivier F, Humberclaude V, Petrof B, Matecki S. Ventilatory parameters and maximal respiratory pressure changes with age in Duchenne muscular dystrophy patients. Pediatr Pulmonol.2010;45(6):552-559.

18. Phillips MF, Quinlivan RC, Edwards RH, Calverley PM. Changes in spirometry over time as a prognostic marker in patients with Duchenne muscular dystrophy. Am J Respir Crit Care Med.2001;164(12):2191-2194. 
19. Finder JD, Birnkrant D, Carl J, et al. Respiratory care of the patient with Duchenne muscular dystrophy: ATS consensus statement.Am J Respir Crit Care Med. 2004;170(4):456-465.

20. Finder J, Mayer OH, Sheehan D, et al. Pulmonary Endpoints in Duchenne Muscular Dystrophy. A Workshop Summary. Am J Respir Crit Care Med. 2017;196(4):512-519.

21. Rideau Y, Jankowski LW, Grellet J. Respiratory function in the muscular dystrophies. Muscle Nerve. 1981;4(2):155-164.

22. Birnkrant DJ, Bushby K, Bann CM, et al. Diagnosis and management of Duchenne muscular dystrophy, part 2: respiratory, cardiac, bone health, and orthopaedic management. Lancet Neurol. 2018;17(4):347-361.

23. Birnkrant DJ, Ashwath ML, Noritz GH, et al. Cardiac and pulmonary function variability in Duchenne/Becker muscular dystrophy: an initial report. J Child Neurol. 2010;25(9):1110-1115.

24. McKim DA, Road J, Avendano M, et al. Home mechanical ventilation: a Canadian Thoracic Society clinical practice guideline. Can Respir J. 2011;18(4):197-215.

25. Molgat-Seon Y, Hannan LM, Dominelli PB, et al. Lung volume recruitment acutely increases respiratory system compliance in individuals with severe respiratory muscle weakness. ERJ Open Res. 2017;3(1).

26. McKim DA, Katz SL, Barrowman N, Ni A, LeBlanc C. Lung volume recruitment slows pulmonary function decline in Duchenne muscular dystrophy. Arch Phys Med Rehabil. 2012;93(7):1117-1122.

27. Connolly AM, Florence JM, Zaidman CM, et al. Clinical trial readiness in non-ambulatory boys and men with duchenne muscular dystrophy: MDA-DMD network follow-up. Muscle Nerve.2016;54(4):681-689.

28. Katz SL, Barrowman N, Monsour A, Su S, Hoey L, McKim D. Long-Term Effects of Lung Volume Recruitment on Maximal Inspiratory Capacity and Vital Capacity in Duchenne Muscular Dystrophy. Ann Am Thorac Soc.2016;13(2):217-222.

29. Rall S, Grimm T. Survival in Duchenne muscular dystrophy. Acta Myol. 2012;31(2):117-120.

30. Passamano L, Taglia A, Palladino A, et al. Improvement of survival in Duchenne Muscular Dystrophy: retrospective analysis of 835 patients.Acta Myol. 2012;31(2):121-125.

31. Ishikawa Y, Miura T, Ishikawa Y, et al. Duchenne muscular dystrophy: survival by cardio-respiratory interventions. Neuromuscul Disord.2011;21(1):47-51.

32. McKim DA, Griller N, LeBlanc C, Woolnough A, King J. Twenty-four hour noninvasive ventilation in Duchenne muscular dystrophy: a safe alternative to tracheostomy. Can Respir J. 2013;20(1):e5-9.

33. Toussaint M, Steens M, Wasteels G, Soudon P. Diurnal ventilation via mouthpiece: survival in end-stage Duchenne patients. Eur Respir J. 2006;28(3):549-555.

34. Kleopa KA, Sherman M, Neal B, Romano GJ, Heiman-Patterson T. Bipap improves survival and rate of pulmonary function decline in patients with ALS. J Neurol Sci. 1999;164(1):82-88.

35. Toussaint M, Soudon P, Kinnear W. Effect of non-invasive ventilation on respiratory muscle loading and endurance in patients with Duchenne muscular dystrophy. Thorax. 2008;63(5):430-434.

36. Schonhofer B, Wallstein S, Wiese C, Kohler D. Noninvasive mechanical ventilation improves endurance performance in patients with chronic respiratory failure due to thoracic restriction. Chest.2001;119(5):13711378 .

37. Nickol AH, Hart N, Hopkinson NS, Moxham J, Simonds A, Polkey MI. Mechanisms of improvement of respiratory failure in patients with restrictive thoracic disease treated with non-invasive ventilation. Thorax. 2005;60(9):754-760.

38. Gomez-Merino E, Bach JR. Duchenne muscular dystrophy: prolongation of life by noninvasive ventilation and mechanically assisted coughing.Am J Phys Med Rehabil. 2002;81(6):411-415. 
39. Stehling F, Bouikidis A, Schara U, Mellies U. Mechanical insufflation/exsufflation improves vital capacity in neuromuscular disorders. Chron Respir Dis. 2015;12(1):31-35.

40. Neve V, Cuisset JM, Edme JL, et al. Sniff nasal inspiratory pressure in the longitudinal assessment of young Duchenne muscular dystrophy children. Eur Respir J. 2013;42(3):671-680.

41. Mercuri E, Muntoni F, Osorio AN, et al. Safety and effectiveness of ataluren: comparison of results from the STRIDE Registry and CINRG DMD Natural History Study. J Comp Eff Res. 2020;9(5):341-360.

42. Jin JB, Carter JC, Sheehan DW, Birnkrant DJ. Cardiopulmonary phenotypic discordance is common in Duchenne muscular dystrophy.Pediatr Pulmonol. 2019;54(2):186-193.

43. Magri F, Govoni A, D'Angelo MG, et al. Genotype and phenotype characterization in a large dystrophinopathic cohort with extended follow-up. J Neurol. 2011;258(9):1610-1623.

44. Roy B, Friesen WJ, Tomizawa Y, et al. Ataluren stimulates ribosomal selection of near-cognate tRNAs to promote nonsense suppression.Proc Natl Acad Sci U S A. 2016;113(44):12508-12513.

45. Khan N, Eliopoulos H, Han L, et al. Eteplirsen Treatment Attenuates Respiratory Decline in Ambulatory and Non-Ambulatory Patients with Duchenne Muscular Dystrophy. J Neuromuscul Dis.2019;6(2):213-225.

46. Buyse GM, Goemans N, van den Hauwe M, Meier T. Effects of glucocorticoids and idebenone on respiratory function in patients with duchenne muscular dystrophy. Pediatr Pulmonol.2013;48(9):912-920.

47. Buyse GM, Voit T, Schara U, et al. Efficacy of idebenone on respiratory function in patients with Duchenne muscular dystrophy not using glucocorticoids (DELOS): a double-blind randomised placebo-controlled phase 3 trial. Lancet.2015;385(9979):1748-1757.

48. Birnkrant DJ, Ararat E, Mhanna MJ. Cardiac phenotype determines survival in Duchenne muscular dystrophy. Pediatr Pulmonol.2016;51(1):70-76.

49. Vincken WG, Elleker MG, Cosio MG. Flow-volume loop changes reflecting respiratory muscle weakness in chronic neuromuscular disorders. Am J Med. 1987;83(4):673-680.

50. Hahn A, Bach JR, Delaubier A, Renardel-Irani A, Guillou C, Rideau Y. Clinical implications of maximal respiratory pressure determinations for individuals with Duchenne muscular dystrophy. Arch Phys Med Rehabil. 1997;78(1):1-6.

51. Nicot F, Hart N, Forin V, et al. Respiratory muscle testing: a valuable tool for children with neuromuscular disorders. Am J Respir Crit Care Med. 2006;174(1):67-74.

52. Inacio MC, Chen Y, Paxton EW, Namba RS, Kurtz SM, Cafri G. Statistics in Brief: An Introduction to the Use of Propensity Scores. Clin Orthop Relat Res. 2015;473(8):2722-2726.

53. Kempf L, Goldsmith JC, Temple R. Challenges of developing and conducting clinical trials in rare disorders. Am J Med Genet A.2018;176(4):773-783. 